\title{
90. Electron-microscopic Observations on Nuclear Exchange during Conjugation in Paramecium multimicronucleatum
}

\author{
By Fumie Inaba, Kikuko Imamoto, \\ and Yoshiko Suganuma \\ Department of Zoology, Faculty of Science, \\ Nara Women's University \\ (Comm. by Taku KomaI, M.J.A., April 12, 1966)
}

Recently, morphological details of the region of contact between conjugating individuals of ciliates have become better known by electron-microscopic studies. ${ }^{1-5)}$ However, many problems remain unsolved. One problem is how the pronucleus migrates into the opposite member of the pair. No previous worker has given a detailed description of this process. This report presents results of our electron-microscopic studies designed specifically to elucidate the mode of exchange of pronuclei during conjugation in Paramecium multimicronucleatum.

The materials used were Paramecium multimicronucleatum $\mathrm{CH}$ 323 (mating type III) and CH 326 (mating type IV). Stock cultures were maintained by the procedure reported by Miyake. ${ }^{6}$ Cultures in the logarithmic phase of growth had been maintained for 1-2 days without food. Then the paramecia of the complementary mating types were mixed. Soon after mixing, agglutination of individuals occurred, and pairs were formed in about one hour. The paired animals remained for 12 hours at $25^{\circ} \mathrm{C}$ and then separated.

For electron microscopy, pairs of paramecia were collected at 60 minute intervals from the beginning to the end of pairing, and concentrated in the bottom of a small tube by slight centrifugation. They were fixed for 60 minutes in $1 \% \quad \mathrm{OsO}_{4}$, adjusted to $\mathrm{pH} 7.9$ with veronal acetate buffer. Then they were dehydrated with ethanol and embedded in epoxy resin by Luft's method. ${ }^{7)}$ Sections were cut, mounted on formvar-coated grids and stained with uranyl acetate and lead nitrate. Observations were made with a Model JEM-7 electron microscope.

Figs. 1 and 2 show the transverse sections of the region of contact between conjugating paramecia in which nuclear exchange appears to be just beginning. The boundary line $(b)$ between the conjugats is composed of two parallel membranes, each $30 \mathrm{~m} \mu$ in thickness, separated by an interspace about $30 \mathrm{~m} \mu$ wide. It is interrupted at places (Fig. 1, i), where the two membranes, one from 


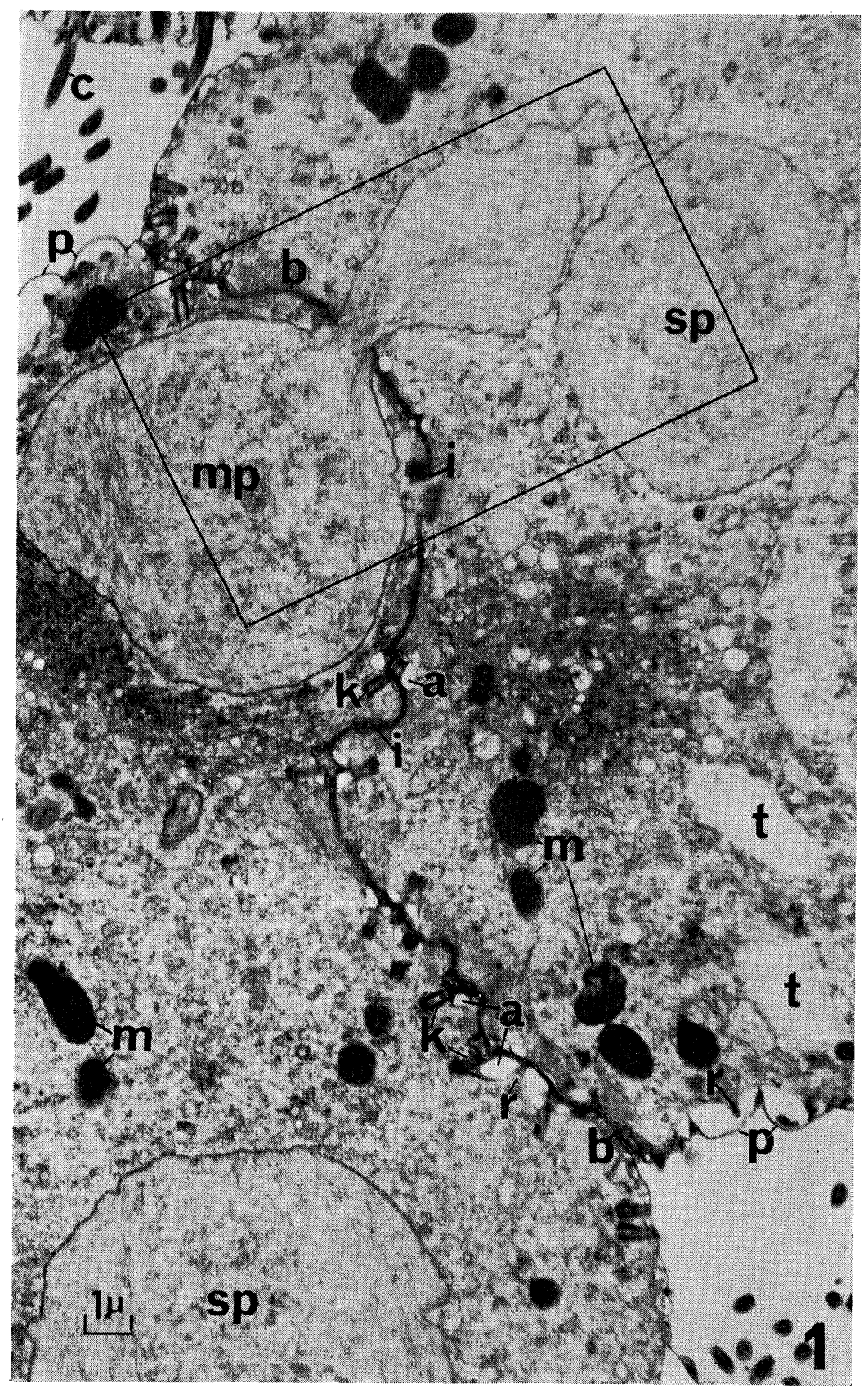

Fig. 1. Electron micrograph of a transverse section through the region of contact of conjugating Paramecium multimicronucleatum. Two pronuclei are observed in the conjugant on the left side, one of which (migratory pronucleus, $m p$ ), is migrating by amoeboid movement and approaching the stationary pronucleus $(s p)$ in the conjugat on the right side. Cilia and trichocysts have disappeared in the contact region, but kinetosomes remain. 


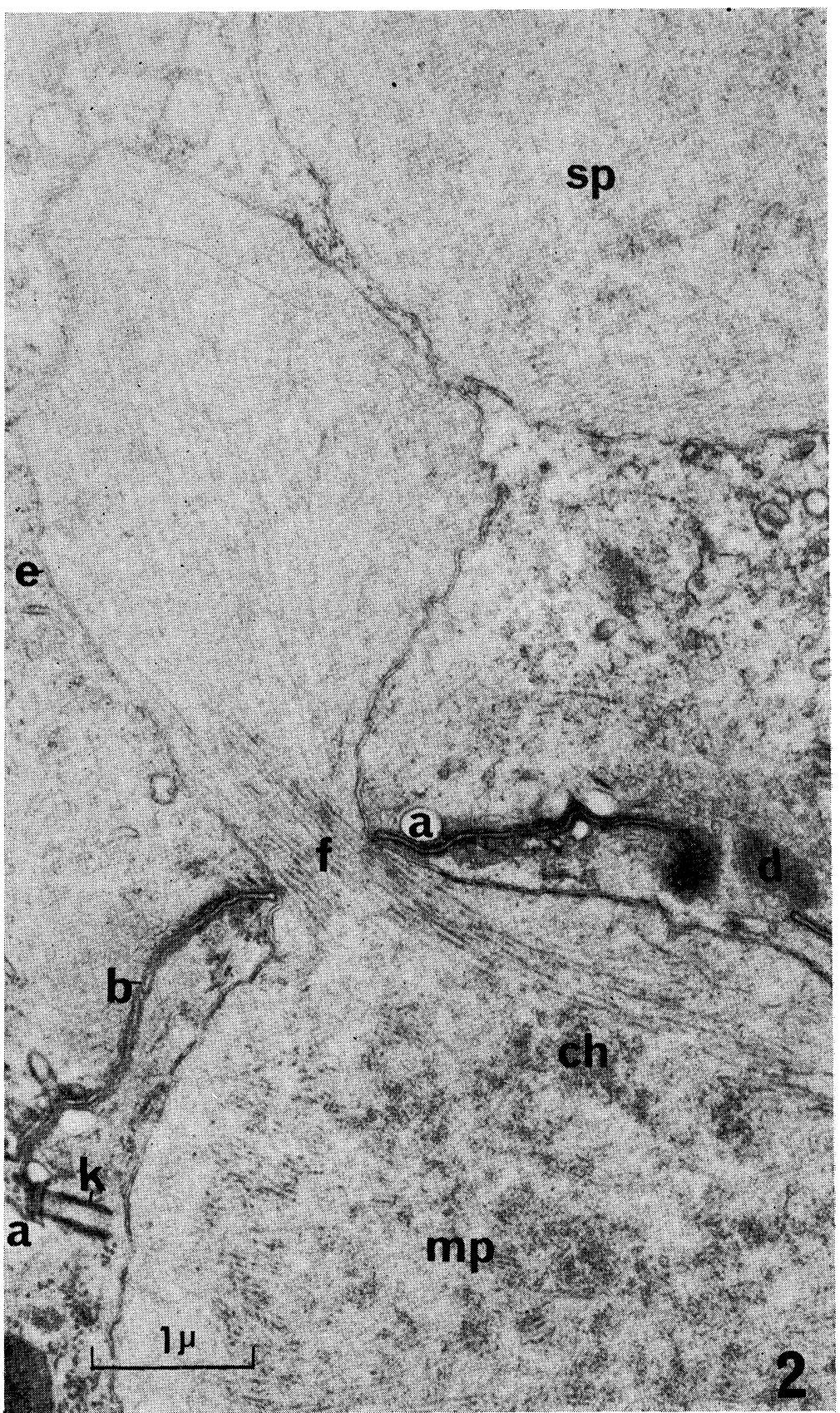

Fig. 2. More enlarged micrograph of a framed portion of Fig. 1. Note a bundle of fibrils extending from the migratory pronucleus $(m p)$ to the pseudopodial extension, which are continuous with the chromonemata.

$a$, sub-pellicular alveolus; $b$, boundary membrane; $c$, cilium; $c h$, chromonema; $d$, dense material; $e$, nuclear envelope; $f$, fibrils of chromonemata; $i$, interruption of the boundary membrane; $k$, kinetosome; $m$, mitochondrion; $m p$, migratory pronucleus; $p$, pellicle; $r$, ectoplasmic ridge; $s p$, stationary pronucleus; $t$, trichocyst. 
each conjugat, are joined together. The cytoplasmic communication between the pair occurs at these places. The pronucleus $(m p)$ in the member on the left side of the figure is in the course of migration through one of these communicating regions. The diameter of the migrating pronucleus is about $8 \mu$, twice as large as that of the vegetative micronuclei, while the passage is only $1 \mu$ in width. Migration is apparently carried out in amoeboid fashion. A part of the migratory pronucleus has already penetrated into the cytoplasm of the opposite member of the pair as a pseudopodial extension, approaching the nucleus, presumably a stationary pronucleus $(s p)$ in the other member. The boundary line is pressed out by the migratory pronucleus, appearing as a curved line, and the cytoplasm around the pronucleus appears to have increased in density. A bundle of fine fibrils (Fig. $2, f$ ) is stretched through the constriction from the main body of the pronucleus to the pseudopodial extension. The fibrils, which are each about $40 \mathrm{~m} \mu$ in diameter and composed of pairs of parallel fibrils, are probably straightened chromonemata. They are continuous with the fibrils in the coiled threads about $300 \mathrm{~m} \mu$ thick (Fig. 2, ch) extending throughout the pronucleus. Cilia have snapped off at the base, but kinetosomes $(k)$ persist in the ectoplasm beneath the boundary line. Small alveoli $(a)$ are observed adjacent to the kinetosome. These are residues of the sub-pellicular alveoli, which originally surrounded the ectoplasmic ridge (Fig. 1, r) as seen in the verge of the contact area. Probably the boundary line has been established by flattening of the cortical ridges with simultaneous decrease in the size of the subpellicular alveoli. Trichocysts have disappeared in the contact region. Elliott and Tremor, ${ }^{1)}$ studying the cortical structure of conjugating Tetrahymena pyriformis, observed a single membrane in the area of contact, which they supposed to be formed by fusion of the two surface pellicles; they also found many pores in the membrane connecting the two cells. As these pores are approximately equal in diameter $(0.2 \mu)$ with ciliary openings through which the cilia grew, the authors suggested that these pores were the results of fusion of ciliary openings in the pellicle, and were responsible for the interchange of cytoplasmic particulate material of up to $0.2 \mu$ in diameter. Their photographs of longitudinal sections of the contact region show that the boundary line enclosing the pronucleus of one member of the pair has projected deep into the cytoplasm of the other member, but there is no sign indicating nuclear exchange.

Vivier and Andrét) studied the detailed morphology of the outer pellicle in the contact region of conjugating Paramecium caudatum 
and found cytoplasmic communications at the top of the ectoplasmic ridges. According to the authors, both cilia and trichocysts have disappeared but kinetosomes persist. The same authors ${ }^{5)}$ also observed the migratory pronuclei just preceding the migration lay close to the irregular outline of the boundary line. These pronuclei had an unusual, indented outline and pseudopodia extending in various directions. Although they failed to demonstrate the pronucleus at the exact moment of migration, they assume that the pronucleus becomes narrower and passes through one of the cytoplasmic communications.

The results obtained in the present study provide a clear evidence that the pronucleus migrates into the opposite member of a pair by amoeboid movement. However, the pronucleus is not deformed as strikingly as in $P$. caudatum. The pronucleus maintains its spherical shape except for its pseudopodial extension. It seems likely that the pronucleus migrates by active movement, protruding a pseudopodium in amoeboid fashion, but some other factors such as the presence of a cytoplasmic current might also assist the migration.

With regard to the establishment of a binding area, the observations reported here are in agreement with the findings in $P$. caudatum that cytoplasmic communications are built by fusion at the top of ectoplasmic ridges. The electron-dense material observed in the area of communication may well be dissolution products of the pellicular membrane.

\section{References}

1) Elliott, A. M., and Tremor, J. W. (1958): The fine structure of the pellicle in the contact area of conjugating Tetrahymena pyriformis. J. Biophys. Biochem. Cytol., 4, 839-840.

2) Ehret, C. F., and Powers, E. L. (1959): The cell surface of Paramecium. Intern. Rev. Cytol., 8, 97-133.

3) Schneider, L. (1960): Die Auflösung und Neubildung der Zellmembran bei der Konjugation von Paramecium. Naturwissenschaften, 47, 543-544.

4) Vivier, E., et André, J. (1961): Données structurales et ultrastructurales nouvelles sur la conjugaison de Paramecium caudatum. J. Protozool., 8, $416-426$.

5) André, J., et Vivier, E. (1962): Quelques aspects ultrastructuraux de l'échange micronucléaire lors de la conjugaison chez Paramecium caudatum. J. Ultrastructure Research, 6, 390-406.

6) Miyake, A. (1964): Induction of conjugation by cell-free preparations in Paramecium multimicronucleatum. Science, 146, 1583-1585.

7) Pearse, D. C. (1960): Histological Techniques for Electron Microscopy. Academic Press, New York, p. 84. 